\title{
APRENDIZAGEM COOPERATIVA NO ENSINO DA MODELAGEM DA INFORMAÇÃO DA CONSTRUÇÃO NO CONTEXTO DO ISOLAMENTO SOCIAL
}

Ricardo Albuquerque de Sousa - ricardosousa@ alu.ufc.br Camily Vasconcelos Barbosa - vasconcelosmily@alu.ufc.br João Hudson Oliveira Nunes - joaohudson001 @ gmail.com Cely Martins Santos de Alencar - celyms@ufc.br Departamento de Integração Acadêmica e Tecnológica - DIATEC Universidade Federal do Ceará, Campus do Pici, s/n- Bloco 710 60455-760 - Fortaleza - CE

Resumo: O presente artigo teve como objetivo analisar a dinâmica de funcionamento de grupos de estudos de projetos de instalações utilizando-se de ferramentas BIM sob a ótica da aprendizagem cooperativa. Devido ao isolamento social causado pelo Covid-19, houve a necessidade de realizar as atividades a distância por meio de plataformas digitais, havendo assim uma mudança nos meios de interação do grupo. Com auxílio de embasamento teórico sobre a aprendizagem cooperativa e ferramentas digitais a análise do desenvolvimento dos grupos sucedeu-se por meio de resultados e percepções individuais quanto ao aprendizado possibilitado pelo grupo e à metodologia utilizada no desenvolvimento do mesmo. Dessa forma, foi possível observar características inerentes a aprendizagem cooperativa tais como responsabilidade individual e heterogeneidade. Entretanto, alguns atributos não foram contemplados, tais como o desenvolvimento de habilidades interpessoais e interdependência positiva. Com isso, mudanças na formação e no funcionamento do grupo são sugeridas a fim de melhorá-lo.

Palavras-chave: Aprendizagem cooperativa. Grupo de estudos. Ensino a distância. BIM. 


\section{INTRODUÇÃO}

As perceptíveis mudanças nos meios de comunicação ocorridas nas últimas duas décadas abrem espaço para novas formas de interação que, aliadas a metodologias de aprendizagem colaborativa já existentes, podem funcionar como uma saída viável para enfrentar dificuldades recorrentes e também excepcionais.

O presente artigo visa analisar a dinâmica de funcionamento de grupos de estudos da disciplina de Modelagem da Informação da Construção (MIC) voltada para instalações prediais. Grupos esses inseridos como parte das atividades do Escritório de Projetos Integrados de Engenharia (EPE), projeto de extensão da Universidade Federal do Ceará (UFC). No contexto de um projeto de extensão alguns problemas específicos serviram de motivação para utilização desse modelo de aprendizagem colaborativa em células de estudo, tais como a necessidade de capacitação dos membros e a baixa motivação dos membros em trabalhos individuais.

Além disso, logo após estruturação dos grupos e direcionamentos dos membros para disciplinas de elétrica e hidrossanitário, um novo entrave fez com que a ideia inicial de encontros físicos e interação direta fosse revista. De fato, no início de março de 2020 a Universidade Federal do Ceará interrompeu suas atividades face aos primeiros casos de covid-19. Apesar da interrupção de aulas presenciais o EPE continuou com atividades de maneira remota, de modo que por meio de ferramentas online os grupos de estudo foram mantidos.

Desse modo, o estudo tem como objetivo principal analisar o formato escolhido para aprendizagem de tecnologia BIM aplicada em projetos de engenharia civil. São visados os seguintes objetivos específicos: analisar qualitativamente a interação dentro dos grupos e identificar melhorias necessárias para posterior aplicação com novos membros.

Para tanto a metodologia adotada conta inicialmente com a alocação de membros para compor os grupos e o estruturação de cronograma e material pedagógico inicial. Em seguida elencou-se bibliografia existente (artigos, livros, projetos de aprendizagem cooperativa, etc.) sobre aprendizagem cooperativa e ensino mediado por tecnologia. De modo que, tendo base teórica suficiente, busca-se identificar pontos importantes, tanto positivamente como negativamente, por meio da análise de interações entre membros (nas diferentes plataformas digitais utilizadas), resultados e percepções individuais. Cabe ressaltar que as percepções individuais dos membros se tratam de parâmetros de origem subjetiva e, portanto, é necessário reconhecer as limitações de se trabalhar com esse tipo de informação.

\section{FUNDAMENTAÇÃO TEÓRICA}

\subsection{Aprendizagem cooperativa}

Aprendizagem colaborativa, caracterizada por Dillenbourg (1999) como uma situação de aprendizagem na qual duas ou mais pessoas aprendem ou tentam aprender algo juntas, não é algo novo. Também deve-se ter em vista as possibilidade intrínsecas a essa modalidade que, na perspectiva de Campos et al. (2003, p.26, apud CASTILHO et al., 2014, p. 54), "é uma técnica ou proposta pedagógica na qual estudantes ajudam-se no processo de aprendizagem, atuando como parceiros entre si e com o professor, com o objetivo de adquirir conhecimento sobre um dado objeto."

Nas palavras de Gerdy (1998, apud WIERSEMA, 2000, p. 3) “o aprendizado é aprimorado quando se parece mais com um esforço de equipe do que com uma corrida solo. [...] . Compartilhar as ideias de uma pessoa e responder às dos outros melhora o pensamento e aprofunda a compreensão." Cabe ressaltar que eventualidades podem causar atrasos de 
cronograma e, nesse sentido, o trabalho em equipe funciona como apoio para continuidade de atividades, algo bem exemplificado na própria Bíblia:

Melhor é serem dois do que um, porque têm melhor paga do seu trabalho. Porque se um cair, o outro levanta o seu companheiro; mas ai do que estiver só; pois, caindo, não haverá outro que o levante. Também, se dois dormirem juntos, eles se aquentarão; mas um só, como se aquentará? E, se alguém prevalecer contra um, os dois lhe resistirão; e o cordão de três dobras não se quebra tão depressa. (Eclesiastes 4:9-12)

Johnson e Johnson (1999) definem que para uma atividade ser cooperativa, cinco princípios básicos devem ser atendidos, que são: Independência Positiva; Responsabilidade Individual; Interação promotora; Habilidades sociais; Processamento de grupo.

- Interdependência Positiva é o senso que todos precisam dos outros para serem alcançar a meta coletiva. Ela é estabelecida pela divisão de tarefas e de materiais com metas de aprendizagens mútuas. Assim, caso haja dificuldades no aprendizado de um, outro deve estar preparado para supri-las.

- Responsabilidade individual trata que a performance individual deve ter o devido valor, pois o resultado coletivo não é o único objetivo, pois o trabalho cooperativo também visa tornar cada indivíduo mais forte.

- Interação promotora diz respeito à colaboração mútua entre os membros. Essa iniciativa visa que a promoção do sucesso individual dos outros membros, por meio de ajuda, suporte, encorajamento, seja a maneira para valorizar os esforços.

- Habilidades Sociais são as ferramentas que o grupo deve possuir, como liderança, tomada de decisões, confiança, comunicação e gerenciamento de conflitos, para que a equipe saiba trabalhar de forma organização, mantendo um alto padrão de aprendizagem, além de manter um convívio harmonioso.

- O processamento de grupo é o gerenciamento das metas que eles desejam alcançar. É a descrição das ações individuais que ajudaram e prejudicaram a organização, o convívio, as metas pretendidas.

Entender estes cinco pilares é importante para que grupo de aprendizagem cooperativa consiga atingir suas metas, o aprendizado seja maximizado e que o grupo trabalhe da melhor forma possível.

Além disso, nem todos os grupos de estudos são cooperativos, conforme Johnson e Johnson (1999). Por exemplo, os tradicionais grupos formados aleatoriamente por professores algumas vezes facilitam a aprendizagem, em outras até impedem o aprendizado e/ou criam desarmonia e frustração. Tomando como base as ideias apresentadas por Johnson e Johnson, Freitas e Freitas (2003, p. 37, apud TORRES; ILARA, 2014, p. 66) apresentam pontos importantes para diferir grupos de trabalho cooperativo e grupos tradicionais, identificando características inerentes aos pilares da aprendizagem cooperativa. Essas características estão presentes no Quadro 1.

Quadro 1 - Características dos grupos de aprendizagem cooperativa

\begin{tabular}{|c|c|}
\hline Interdependência positiva & Preocupação com aprendizagem dos outros \\
\hline Responsabilidade individual & Enfase na tarefa e na sua manutenção \\
\hline Heterogeneidade & Ensino direto dos skills sociais \\
\hline
\end{tabular}


"Os desafios para formar hoje o engenheiro do amanhã"

\begin{tabular}{|c|c|}
\hline Liderança partilhada & Papel do professor: observa e intervém \\
\hline Responsabilidade mútua partilhada & O grupo acompanha sua produtividade \\
\hline
\end{tabular}

Fonte: adaptado de Freitas e Freitas (2003, p. 37, apud TORRES; IRALA, 2015, p. 66)

\subsection{Mediação por meios digitais}

Um grupo virtual é constituído, segundo Lévy (2000, p. 127, apud SCHERER; BRITO, 2014, p. 55), "sobre projetos mútuos, em um processo de cooperação ou de troca, tudo isso independentemente das proximidades geográficas e das filiações institucionais." Se trata de um curso de reciprocidades e compartilhamentos de aprendizados que ultrapassa barreiras geográficas e permite a construção do conhecimento. Nessa perspectiva, as ferramentas digitais são as alternativas adotadas para manutenção e estímulo de colaboração entre os participantes. Não deve se perder de perspectiva que os atores principais continuam a ser os participantes de modo que os meios virtuais "dependem da atitude desses frente às propostas de ações nos ambientes virtuais de aprendizagem.” (SCHERER; BRITO, 2014, p.54).

Além disso, devem existir mecanismos que visem a continuidade das atividades pois se tratam de realidades e disposições diferentes. O papel do tutor deixa de ser o principal e passa a ser o de coadjuvante, um coadjuvante sempre atento que acompanha e pontua quando necessário, visando sempre garantir aprendizagem e continuidade do processo. $\mathrm{Na}$ modalidade de ensino a distância o professor funciona, de acordo com Oliveira e Silveira (2014, p.97-98, apud NASCIMENTO; SILVA, 2018, p. 77), "como um colaborador ou um intermediador do aprendizado, que deve monitorar e acompanhar o estudante durante todo o processo, até que este consiga se tornar mais proativo e autônomo [...]."

Para facilitar a interação entre os participantes em ambientes virtuais são utilizadas diversas ferramentas. Essas ferramentas podem ser divididas de maneira mais ampla em ferramentas síncronas e assíncronas. Onde as síncronas compreendem aquelas cuja proposta é a comunicação em tempo real, ou seja, membros conectados ao mesmo tempo. As ferramentas assíncronas, contrariamente, dizem respeito às que não requerem interação em tempo real. Nascimento e Silva (2018) com base em pesquisa bibliográfica levantaram ferramentas utilizadas nestes ambientes, sendo estas: material didático-pedagógico; diário; wiki; chat; portfólio.

\section{METODOLOGIA}

A organização utilizada nos grupos de estudos se deu da seguinte forma: inicialmente foi feito um planejamento sobre o que deveria ser estudado além do levantamento de materiais necessários para a execução, reunidos dentro de um cronograma em formato de roteiro. Em seguida, começou-se a se colocar em prática o que foi planejado, assim cada integrante estudava e colocava o assunto em prática. As dúvidas que surgiam eram compartilhadas em grupo de mensageiro eletrônico (WhatsApp), também por meio de reuniões semanais nas quais era relatado o progresso do grupo e compartilhar conhecimentos.

Os dois grupos de estudos analisados estavam voltados para disciplinas distintas, elétrica e hidráulica. O primeiro, de elétrica, composto por três membros, sendo um deles responsável por disponibilizar inicialmente cronograma e materiais e acompanhar o andamento das atividades. O segundo grupo funcionou de forma parecida, também composto por três membros. Os grupos não possuíam membros em comum, mas optou-se por identificá-los genericamente por membro 1 , membro 2 e membro 3. A definição do cronograma e etapas macro dos estudos e desenvolvimento de projetos foi feita por meio de consulta a membros 
mais familiarizados com as disciplinas. Esses membros estiveram presentes ao longo das atividades, inclusive participando dos grupos de interação criados.

O ambiente de comunicação dos dois grupos foi majoritariamente por aplicativo de mensagens (WhatsApp), de modo que cada núcleo possuía um grupo no mensageiro. No grupo de estudos de elétrica algumas reuniões de acompanhamento foram realizadas como forma de expressar dúvidas e seguir o cronograma. Com o tempo, no entanto, o membro responsável pelo acompanhamento macro opto por mensagens particulares instigando membros a respeito de observações pontuais. Dinâmica semelhante seguiu o grupo de hidráulica, com algumas particularidades inerentes a administração macro realizada por um membro diferente.

Ambos também contaram com uma pasta virtual (google drive) com os projetos desenvolvidos, material de estudo inicial e um arquivo escrito construído pelos membros (manual/roteiro para execução do projeto). Cabe ressaltar que todos tinham a oportunidade de incluir novos arquivos e editar os existentes, de fato eram encorajados. A pasta também funcionava como meio para acompanhamento macro do grupo, visto que permitia verificar atualizações.

Como primeiro instrumento de pesquisa, utilizou-se a coleta de respostas com auxílio de formulário, que era constituído de quatro partes principais: facilidade das etapas do projeto; satisfação pelos resultados de projeto alcançados; indicadores de frequência; percepções gerais sobre o resultado final. Além disso, o formulário apresentava perguntas com espaço para resposta dissertativa sobre a eficácia do formato grupo de estudos frente aos outros métodos de aprendizagem e sugestões

Com exceção das perguntas dissertativas todas os demais itens contidos no formulário permitiam respostas com gradação de intensidade bem definida de modo que para cada resposta foi atribuído um valor variando de 1 a 5 para identificação da tendência. Os dados de dificuldade e satisfação foram analisados por meio de médias simples apresentadas em gráfico de linha como forma de balizar futuros cronogramas, enquanto os dados de frequência e percepção foram analisados por meio de gráficos tipo barra a fim de conseguir ter uma visão individual dos membros. Deu-se especial atenção para indicadores abaixo de 3, que para frequência e percepção geral foi considerado como insuficiente. Os indicadores foram identificados por valores como indicado no Quadro 2.

Quadro 2 - Correspondência entre respostas e valores adotados

\begin{tabular}{|l|l|l|l|l|l|}
\hline Valor & $\mathbf{1}$ & $\mathbf{2}$ & $\mathbf{3}$ & $\mathbf{4}$ & $\mathbf{5}$ \\
\hline Dificuldade & Muito difícil & Difícil & $\begin{array}{l}\text { Intermediár } \\
\text { io }\end{array}$ & Fácil & Muito fácil \\
\hline Satisfação & Mudaria tudo & $\begin{array}{l}\text { Mudaria muitas } \\
\text { coisas }\end{array}$ & $\begin{array}{l}\text { Mudaria } \\
\text { algumas } \\
\text { coisas }\end{array}$ & $\begin{array}{l}\text { Mudaria } \\
\text { poucas coisas }\end{array}$ & $\begin{array}{l}\text { Não mudaria } \\
\text { nada }\end{array}$ \\
\hline Frequência & Nunca & Quase nunca & $\begin{array}{l}\text { Algumas } \\
\text { vezes }\end{array}$ & Quase sempre & Sempre \\
\hline $\begin{array}{l}\text { Percepção } \\
\text { geral }\end{array}$ & $\begin{array}{l}\text { Discordo } \\
\text { completamente }\end{array}$ & $\begin{array}{l}\text { Discordo, mas } \\
\text { não } \\
\text { completamente }\end{array}$ & $\begin{array}{l}\text { Não tenho } \\
\text { certeza }\end{array}$ & $\begin{array}{l}\text { Concordo, mas } \\
\text { não plenamente }\end{array}$ & $\begin{array}{l}\text { Concordo } \\
\text { plenamente }\end{array}$ \\
\hline
\end{tabular}

Fonte: Elaborado pelos autores 


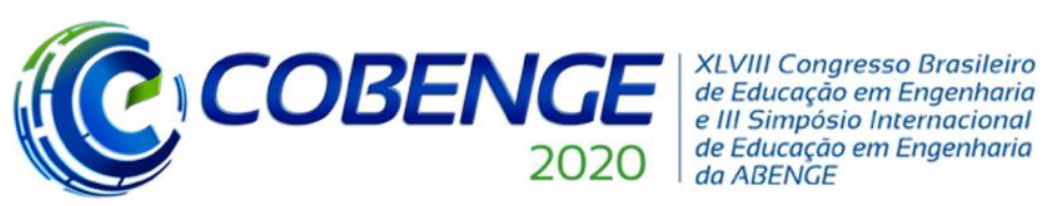

"Os desafios para formar hoje o engenheiro do amanhã"
$\mathrm{Ol} \mathrm{a} \mathrm{O3}$ de dezembro Evento On-line

O segundo instrumento de pesquisa foi a observação direta da frequência de uso e das interações dos membros no grupo de mensagens e a utilização das demais ferramentas virtuais indicadas no Quadro 3, buscando identificar características inerentes a aprendizagem cooperativa, em especial as indicadas no Quadro 1.

Quadro 3 - Tipos de ferramentas de mídia aplicadas

\begin{tabular}{|l|l|}
\hline \multicolumn{1}{|c|}{ Tipo } & \multicolumn{1}{c|}{ Descrição } \\
\hline $\begin{array}{l}\text { Material didático- } \\
\text { pedagógico }\end{array}$ & $\begin{array}{l}\text { Normas; apresentação da disciplina de eletrotécnica } \\
\text { ministrada na UFC; seleção de videoaulas. }\end{array}$ \\
\hline Diário & Controle de atividades por meio de planilha online. \\
\hline Wiki & Manual/roteiro das disciplinas estudadas. \\
\hline Chat & \multicolumn{1}{|c|}{ Grupo em aplicativo de mensagem. } \\
\hline Portfólio & Pastas individuais contendo os trabalhos desenvolvidos. \\
\hline
\end{tabular}

Fonte: Elaborado pelos autores

\section{RESULTADOS E DISCUSSÃO}

Em se tratando de facilidade apenas duas das seis etapas de projeto elétrico e duas das nove etapas de projeto hidrossanitário tiveram média de indicador abaixo de 3 como indica a Figura 1, ou seja, de um modo geral os membros não enfrentaram grandes dificuldades na execução das tarefas previstas. A satisfação pelos resultados obtidos foi elevada, visto que apenas uma etapa de cada grupo apresentou nota 3 e todas as demais ficaram acima desse valor. Cabe ressaltar que maior facilidade não implicou necessariamente maior satisfação pelo resultado como se verifica principalmente no grupo de elétrica comparando a média simples dos indicadores, comportamento que se repete quando se toma os valores por etapa e por membro.

Figura 1 - Média simples dos indicadores de facilidade e satisfação por etapas de projeto Grupo de elétrica

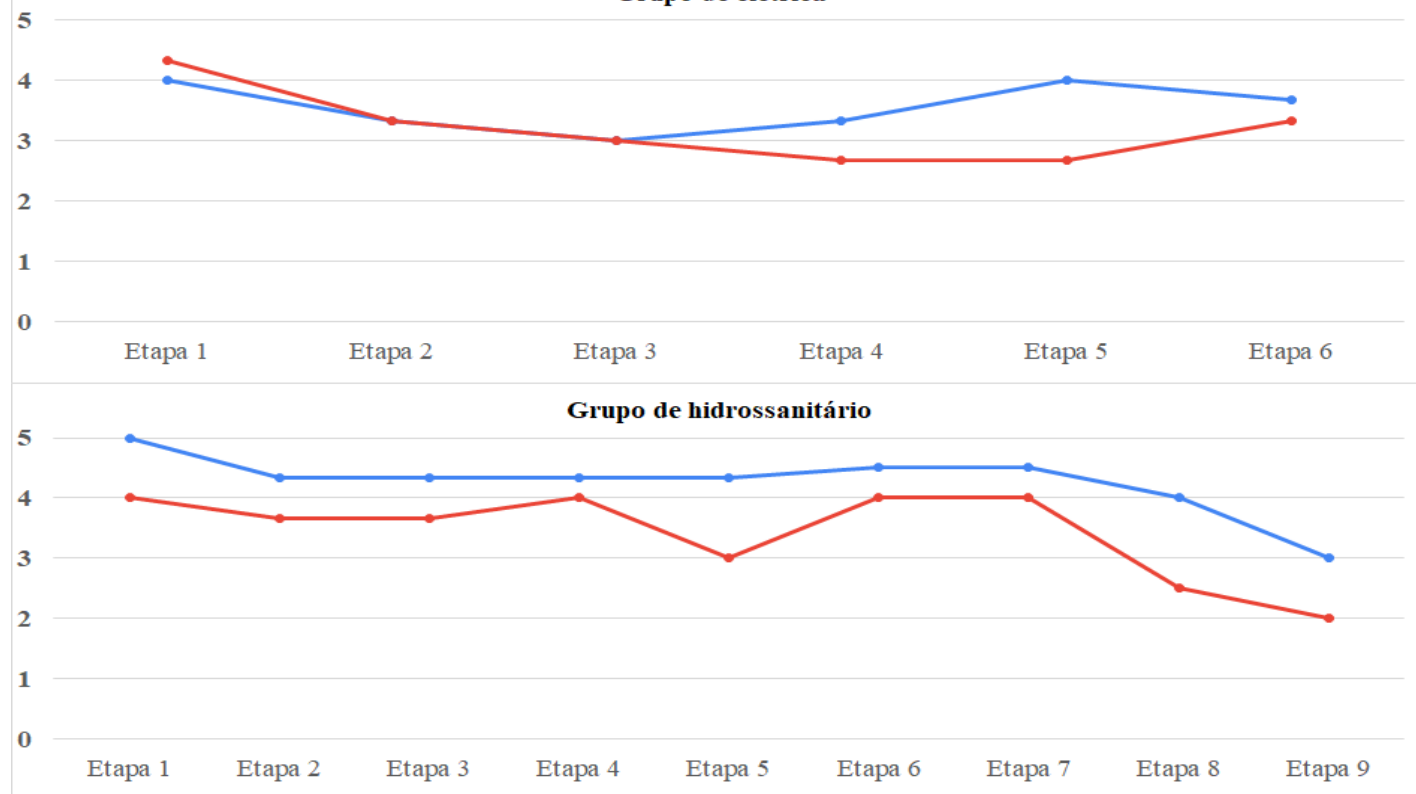

Fonte: Elaborado pelos autores 
(C) COBENCE 2020

"Os desafios para formar hoje o engenheiro do amanhã"
$\mathrm{O1}$ a $\mathrm{O3}$ de dezembro Evento On-line

Apesar dos indicadores de facilidade e satisfação altos alguns membros apresentaram maiores atrasos em virtude da heterogeneidade do grupo em relação a semestre e/ou curso, mas se mantiveram em atividade, indicando que os membros entendiam a importância da responsabilidade individual para o funcionamento saudável do grupo. No entanto, é preciso pontuar que a frequência de atualização de atividades nas pastas de membros esteve condicionada à solicitação direta, mostrando que a responsabilidade individual ainda é passível de melhorias adotando-se, por exemplo, um procedimento de acompanhamento partilhado entre os participantes e não atribuindo isso a um único membro.

É relevante observar que apesar das percepções gerais terem tido valores bem elevados, como indicado na Figura 2 e na Figura 3, pontos ligados a compreensão e autonomia em relação ao processo de projeto apresentaram valores mais baixos. No grupo de elétrica nota-se baixa frequência no uso de materiais escritos e fontes normativas de projeto, enquanto no grupo de hidrossanitário percebe-se que apenas um dos membros buscou frequentemente contribuir com material de apoio. Além disso, verifica-se nos indicadores que a frequência de solicitação de ajuda externa ao grupo foi baixa em ambos. Depreende-se que os grupos ficaram voltados especialmente para replicação de informações provenientes de vídeo aulas ou materiais já disponíveis. A adoção de manuais construídos colaborativamente seria, portanto, uma forma viável de enfrentamento desse problema, como será discutido a seguir.

Figura 2 - Indicadores de frequência e percepção geral, Grupo de Estudos de Elétrica

Frequência
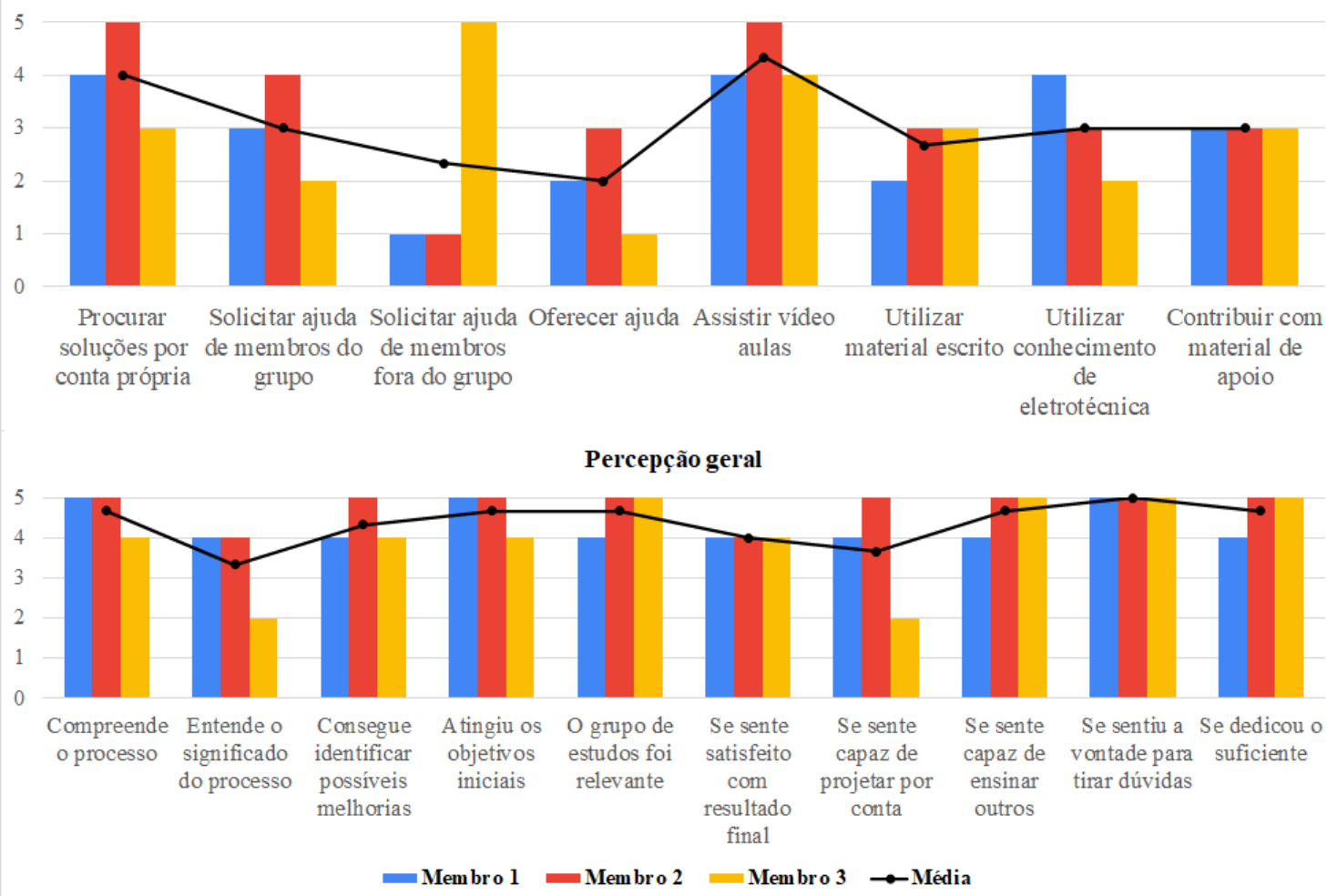

Fonte: Elaborado pelos autores 
Figura 3 - Indicadores de frequência e percepção geral, Grupo de Estudos de Hidrossanitário Frequência
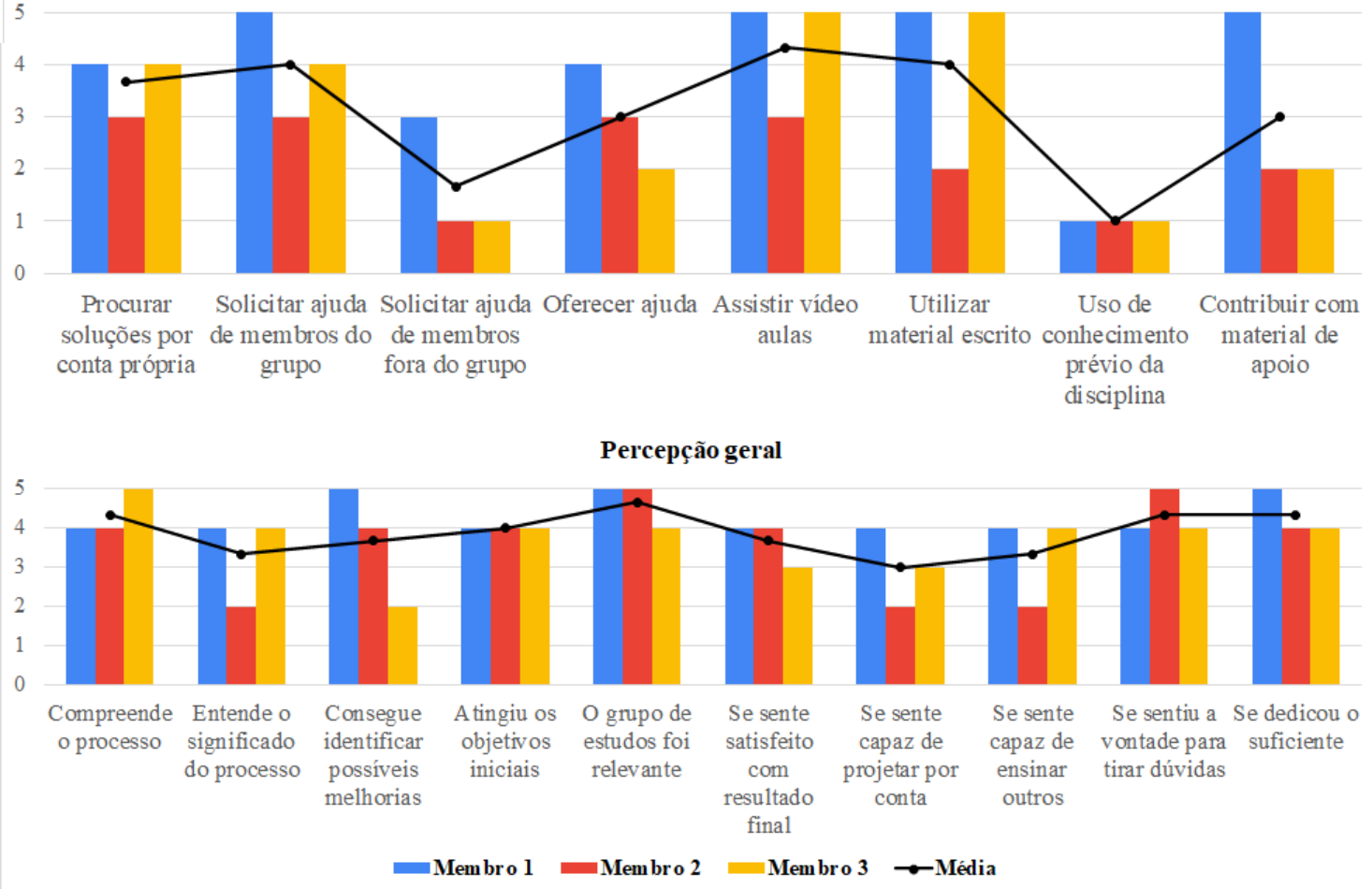

Fonte: Elaborado pelos autores

No caso específico dos manuais de disciplina desenvolvidos pelos membros é interessante notar que os manuais se mostraram uma alternativa importante na construção de conhecimentos mais profundos, visto que suscitou dúvidas que não foram reconhecidas de imediato na execução dos projetos. No entanto, provocou maior engajamento no grupo de elétrica, observação confirmada no Quadro 4 que indica a quantidade de dias nos quais o membro efetuou edições no arquivo de texto desde sua criação. A possível explicação seria o período de início escolhido, pois enquanto no grupo de hidrossanitário o roteiro foi iniciado pelo coordenador desde o começo, no de elétrica apenas após conclusão dos modelos projetados. Também é importante ressaltar que dos três membros do grupo de hidrossanitário, dois não tiveram nenhum contato prévio com a disciplina estudada e isso fica evidenciado pelo baixo uso de conhecimento prévio indicado na Figura 3. De modo que o grupo de hidrossanitário possuía menos autonomia.

Quadro 4 - Quantidade de dias em que o membro fez edições no manual/roteiro

\begin{tabular}{|l|l|l|l|l|l|}
\hline \multicolumn{3}{|c|}{ Manual do grupo de elétrica } & \multicolumn{3}{l|}{ Roteiro do grupo de hidrossanitário } \\
\hline Membro 1 & Membro 2 & Membro 3 & Membro 1 & Membro 2 & Membro 3 \\
\hline 9 dias & 2 dias & 5 dias & 5 dias & 2 dias & 0 dias \\
\hline
\end{tabular}

Fonte: Elaborado pelos autores

Nota-se também que a frequência com que os membros ofereceram ajuda foi médio ou baixo, além da média frequência de contribuição com materiais de auxílio no grupo de elétrica e baixa frequência de contribuição no grupo de hidrossanitário. A análise de discussões nos grupos de mensagens e durante reuniões complementa e confirma o que os indicadores de frequência apontam a esse respeito. De modo que não houve grande preocupação com 
aprendizagem dos outros participantes e que a interdependência positiva dos grupos foi insuficiente.

Ainda a respeito das ferramentas de interação virtual, não foi observado o desenvolvimento da liderança partilhada e do ensino direto de habilidade sociais, isso pode ser explicado pelo tipo de proposta adotado para organização do grupo. Ou seja, o membro responsável pelo planejamento inicial acumulou atividades de acompanhamento e coordenação praticamente por conta própria. Portanto, fica evidenciado que formações futuras devem buscar sobretudo um modelo de partilha de liderança, formato que provavelmente implicará em melhorias tanto no desenvolvimento de habilidades sociais como da interdependência positiva do grupo.

Com a análise dos formulários e também das ferramentas foi possível destacar outras características identificadas nos grupos, tais características estão destacadas em negrito no Quadro 5.

Quadro 5 - Características observadas nos grupos de estudo, em negrito

\begin{tabular}{|c|c|}
\hline Interdependência positiva & Preocupação com aprendizagem dos outros \\
\hline Responsabilidade individual & Ênfase na tarefa e na sua manutenção \\
\hline Heterogeneidade & Ensino direto dos skills sociais \\
\hline Liderança partilhada & Papel do professor: observa e intervém \\
\hline Responsabilidade mútua partilhada & O grupo acompanha sua produtividade \\
\hline
\end{tabular}

Fonte: adaptado de Freitas e Freitas (2003, p. 37, apud TORRES; IRALA, 2015, p. 66)

\section{CONSIDERAÇÕES FINAIS}

O estudo teve como objetivo principal analisar o formato escolhido para aprendizagem de tecnologia BIM aplicada em projetos de instalações. Tal objetivo foi alcançado conjuntamente com os objetivos específicos, ou seja, foi possível analisar o funcionamento dos grupos qualitativamente, além de identificar as mudanças necessárias para posterior aplicação em novos grupos formados

Conforme o exposto, fica evidente que a metodologia adotada pelo grupo de estudos possui como resultado aspectos característicos da aprendizagem cooperativa. No entanto, é evidente que apenas essas características não foram suficientes para manter um funcionamento ideal nos moldes da aprendizagem cooperativa e que, como consequência, as ferramentas digitais adotadas foram subutilizadas.

Tendo isso em vista, alguns atributos precisam ser melhorados a fim de aprimorar a interação dentro do grupo de estudos e alcançar um aprendizado mais aprofundado das disciplinas estudadas. Entre esses atributos ficam em destaque o desenvolvimento de habilidade sociais e a interdependência positiva do grupo, que podem ser alcançados por meio da partilha da liderança dentro do grupo. Além disso, fica evidenciado que ferramentas de construção conjunta como manuais, roteiros e fluxogramas constituem ferramentas relevantes para alcançar maior autonomia e aprendizados aprofundados.

\section{Agradecimentos}

Nosso agradecimento ao Escritório de Projetos Integrados em Engenharia (EPE) que proporcionou e auxiliou o estudo em grupo dos participantes e ao Departamento de Integração Acadêmica e Tecnológica (DIATEC), ao qual o EPE é vinculado. 
"Os desafios para formar hoje o engenheiro do amanhã"

\title{
REFERÊNCIAS
}

CASTILHO, Antonio M. Dias et al. A rede social facebook como ferramenta pedagógica no processo de ensino-aprendizagem de língua inglesa. Revista Transformar, Itaperuna, n. 6, p. 42-63, 2014.

DILLENBOURG, Pierre. What do you mean by collaborative learning?. In: DILLENBOURG, Pierre (Ed.). Collaborative-learning: Cognitive and Computational Approaches. 1 ${ }^{\text {a }}$. ed. Oxford: Elsevier Publishing, 1999. p. 1-19.

JOHNSON, David W.; JOHNSON, Roger T. Making Cooperative Learning Work. Theory Into Practice, v. 38, p. 67-72, 1999.

SCHERER, Suely; BRITO, Glaucia da Silva. Educação a distância: possibilidades e desafios para a aprendizagem cooperativa em ambientes virtuais de aprendizagem. Educar em Revista, Curitiba, n. 4, p. 53-77, 2014.

TORRES, Patrícia Lupion; IRALA, Esrom A. F. prendizagem Colaborativa: Teoria e Prática. In: TORRES, Patrícia Lupion (org.). Complexidade: redes e conexões na produção do conhecimento. $1^{\text {a }}$. ed. Curitiba: SENAR-PR, 2014. p. 61-94.

WIERSEMA, Nico. How does Collaborative Learning actually work in a classroom and how do students react to it? A Brief Reflection. Disponível em: https://www.eric.ed.gov/?q=nico+wiersema\&id=ED464510. Acesso em: 30 mai. 2020.

\section{PROJECT STUDY GROUPS IN THE CONTEXT OF SOCIAL ISOLATION}

\begin{abstract}
The aim of this article was to analyze the dynamics of the functioning of study groups of installation projects using BIM tools from the perspective of cooperative learning. Due to the social isolation caused by Codiv-19, there was a need to carry out activities at a distance through digital platforms, thus changing the means of interaction of the group. With the aid of a theoretical basis on cooperative learning and digital tools, the analysis of the development of the groups took place through results and individual perceptions regarding the learning made possible by the group and the methodology used in the development of the group. Thus, it was possible to observe characteristics inherent to cooperative learning such as individual responsibility and heterogeneity. However, some attributes have not been identified, such as the development of interpersonal skills and positive interdependence. Thus, changes in the formation and functioning of the group are suggested in order to improve it.
\end{abstract}

Keywords: cooperative learning, study group, distance learning, BIM. 